\title{
GAZE AVOIDANCE: PERSONALITY AND SOCIAL JUDGMENTS OF PEOPLE WHO AVOID DIRECT FACE-TO- FACE CONTACT
}

\author{
Randy J. Larsen and Todd K. Shackelford \\ Department of Psychology, University of Michigan, 525 East University, \\ Ann Arbor, MI 48109-1109, U.S.A.
}

(Received 21 February 1996)

\begin{abstract}
Summary-Some people maintain direct, face-to-face contact during interaction, whereas others avert their gaze or turn their face while interacting. Research on individual differences in gaze avoidance, while sparse. falls into two areas. One concerns the personality and psychopathology correlates of gaze aversion, and the other concerns social judgments made of people who avert their gaze during interaction. The findings generally show that gaze aversion is associated with unfavorable traits (shyness, social anxiety, risk for schizophrenia) and negative social evaluations (gaze averse people are rated as more deceptive and less sincere). The present study took advantage of an archival data set that contained facial photographs from which gaze avoidance could be scored. The correlates of gaze avoidance were different for men and women. Gaze avoidant men tended to be emotionally inhibited and overconirolled, and reported a high incidence of various psychosomatic and physical symptoms. Guze avoidant women, on the other hand, were high on measures of psychopathy, hysteria, and traditional femininity, they tended to have fewer physical symptoms. Gaze avoidant women were also viewed by others in a negative light (as being disagreeable, unconscientious, unattractive, and even somewhat lower on intelligence) In males, none of the social judgment variables correlated significantly with gaze avoidance. Results are discussed in terms of sex differences in the meaning and communicative function of this non-verbal social behavior. Copyright 0 1996 Elsevier Science Ltd.
\end{abstract}

\section{INTRODUCTION}

Research on gaze direction and eye contact has established the importance of this behavior for effective social interaction. A direct, face-to-face position, along with eye contact, is an effective way to capture the attention of another person, so much so that this behavior is thought to be a fundamental principle for effective communication (Anolli \& Lambiase, 1990; Argyle, Henderson, Bond \& Iizuka, 1986; Sherrard, 1993). Researchers have documented the role of facial direction and eye contact in greeting ceremonies from diverse cultures (Argyle \& Cook, 1976). Gaze behavior appears to be related to negotiating hierarchical social orders when greeting others. For example, gaze avoidance (turning the face away) during greetings serves to convey status and authority onto the person who is not being looked at (Duranti, 1992). Very similar findings on gaze behaviors have been obtained in studies on non-human primates (de Waal, 1982; Linnankoski, Gronroos \& Pertovaara, 1993). Even in non-primates, a direct gaze can communicate status or threat. Lizards, for example, are more likely to flee if an approaching human is looking directly at them than if the approaching human has his/her face turned away (Burger, Gochfeld \& Murray, 1992). Similar evidence for preferential processing of gaze direction across a variety of species is reviewed by Baron-Cohen (1994).

Research with both human and non-human primates suggests that the ability to discriminate whether another is looking directly at oneself is under control of that portion of the brain responsible for processing facial expressions. Monkeys who were taught a forced-choice discrimination task, in which they learned to detect which of a pair of photographed faces was directly facing the camera (and hence them), lost this ability to discriminate after ablation of the superior temporal sulcus (Campbell, Heywood, Cowey \& Regard, 1990). Two human patients with prosopagnosia* were unable to correctly detect, out of a series of facial photos, which face was facing straight at them (Campbell et al. 1990); normal control $S$ s could easily do this task. Neurologists generally conclude

* Prosopagnosia is an agnosia for faces and facial displays, from the Greek proso. face: a. not: and gnosir, perception). 
that facial perception is neurologically unique, and that the processing of facial stimuli is strongly influenced by the orientation of the face relative to the perceiver, as well as any expressive cues present (Kolb \& Whishaw, 1990).

Individual differences in facial turning and eye contact has also come under the scrutiny of psychologists. Two research literatures have formed around the investigation of individual differences in gaze behavior. One literature emphasizes associated characteristics, such as personality and psychopathology correlates of the tendency to avert one's gaze or turn one's face away during interaction. The other literature concerns social judgments that are made about others who turn askew during interaction. We will briefly review the highlights of these bodies of research.

\section{Personality and psychopathology correlates of gaze avoidance*}

The personality variables most frequently studied as correlates of gaze aversion have to do with shyness and social anxiety. For example, Iizuka (1994) found that shyness was a significant predictor of gaze aversion during a stressful interview. Other researchers have shown that social anxiety is related to spending less time directly facing another during interaction (Farabee, Holcom, Ramsey \& Cole, 1993). Moreover, the effects of social anxiety on diminished social gazing are exacerbated when the other person is known ahead of time to 'disagree' with the socially anxious communicator (Farabee et al. 1993). A study of extraversion showed that extraverts were more likely than introverts to maintain longer gazes while listening during social interaction (Iizuka, 1992a). Studies of emergent leaders show that, in groups of male strangers, those $S$ s who emerged as leaders exhibited a prolonged gaze pattern (Kalma, 1992). From such studies we can conclude that individual differences in such variables as surgency, dominance, and extraversion appear to be related to maintaining a direct facial posture and eye contact during interaction, whereas shyness and social anxiety are related to gaze avoidance.

Psychopathology has also been studied in relation to gaze avoidance. Several studies have been conducted on autism. Typically, these studies compare autistic children with age- and sex-matched non-autistic children in terms of various social behaviors. Several studies report that autistic children show more gaze avoidance, as well as other non-verbal communication deficits, compared to nonautistic children (Adrien, Lenoir, Martineau \& Pcrrot, 1993; Buitelaar, Van Engeland, de Kogel \& de Vries, 1991; Volkmar \& Mayes, 1990).

A related area of study concerns gaze avoidance and adult schizophrenia. Walker and Lewine (1990) identified adult-onset schizophrenics, and obtained home movies of these individuals taken years earlier as pre-schizophrenic children. Similar childhood home movies from non-schizophrenic adults were used as a control group for comparison. All home movies were obtained from infancy to 5 years of age. Raters blind to psychiatric outcome of the children rated the home movies on a number of motor behavior variables, including eye contact and head turning during interaction. Children who later developed adulthood schizophrenia were reliably identified from those who did not. Behaviors that discriminated pre-schizophrenic children from normal children included less eye contact and more head turning (Walker \& Lewine, 1990). Similar results were obtained in a study that also used the method of obtaining home movies of pre-schizophrenic adults (Dequardo \& Tandon, 1994).

Studies have also documented other unfavorable life outcomes that are associated with gaze avoidance. In one study, researchers found that infants who showed more head turning away from their parents during the first month of life showed more problems with social relationships 2 years later (Keller \& Zach, 1993). In another study, infants were observed during their first nursing session during their first day of life (D'Alessio, 1990). The amount the infant looked directly at the mother's face during this first possible face-to-face contact predicted more adequate nursing behavior 55 days later.

In summary, individual differences in gaze behavior, even when obtained at very early ages, are associated with a number of distinct traits and a risk for various unfavorable life outcomes. Gaze avoidance people tend to be more shy and socially anxious, more introverted, less likely to emerge

\footnotetext{
* The term 'gaze avoidance' is used to refer to avoiding the gaze of another by turning the face askew from direct face-toface eye-contact.
} 
as leaders, more at risk for autism and adult schizophrenia, and more at risk for developmental difficulties, particularly in social relationships with parents and others.

\section{Social judgments of gaze avoidance people}

The second area of research in the literature concerns judgments and evaluations made of people who exhibit gaze avoidance. Studies in this area typically take the form of creating videotapes of two people interacting, where different versions of the videotape are made by manipulating how much one participant averts his or her face during the interaction. Ss then view the tapes and make ratings of the participants. The ratings are then analyzed for their relationship to the gaze avoidance conditions. In two such experiments, lizuka (1992b) found that the actor in the gaze avoidance condition was evaluated as more anxious and less sincere, less relaxed, and less dominant than when that same actor displayed more face-to-face interaction. Other researchers have found that people who avert their faces during interaction are though to have lower self-esteem (Droney \& Brooks, 1993). In a study of non-verbal cues of deception (Bond, Omar, Mahmoud \& Bonser, 1990), a large number of $S$ s were videotaped while telling a lie and again while telling the truth. Independent $S$ s viewed the videotapes and made lie detection judgments. These social judgments were highly related to gaze avoidance, with the speaker who averts his or her face being judged as more deceptive. Studies of police have also documented that they interpret gaze avoidance as a cue of deceptiveness (Winkel \& Vrij, 1990).

\section{The present study}

The purpose of the present study is to extend our knowledge of the personality and social judgment correlates of gaze aversion. Other than shyness- and surgency-related variables, we know very little about the personality profiles of people who exhibit gaze avoidance. If a broad sample of personality variables are examined, perhaps other personality correlates would emerge. In terms of psychopathology, we know only that autism and schizophrenia are related to gaze avoidance. Perhaps other pathologies (e.g. depression, anxiety) are related to gaze aversion as well. Similarly, other than deceptiveness and a few other related social judgments (e.g. sincerity), we do not known much about the characteristics attributed to people who avert their gaze.

For the present study we were able to take advantage of two-existing data sets where standardized photographs were obtained on each $S$. Fortunately, the photographs were included along with a large variety of personality and psychopathology variables. We were able to code the photographs for facial posture, and analyze the personality data for differences between those who were looking straight at the camera vs those who were not. Clearly, the idea for this study was post hoc to the actual data collection, and so we had no input on what personality variables were actually measured. However, we did select from the data-set a priori those variables we thought most relevant given the literature. We selected several broad-band personality inventories to explore the personality correlates of gaze aversion. We also examined several measures of physical health and emotional well-being. We examined some standard psychopathology variables to evaluate whether other pathological tendencies are associated with gaze aversion. And finally, we had groups of independent raters evaluate the photographed $S$ s along several evaluative and personality dimensions. This will allow us to examine further the social judgment correlates of gaze aversion.

\section{METHOD}

\section{Subjects}

Two samples of $S$ s were involved in this study. Sample 1 consisted of 41 women and 16 men. Sample 2 consisted of 26 women and 18 men. The samples were from different universities in the midwest portion of the U.S. Both samples were college students enrolled in a semester-long independent study course. The course was widely advertised across campus as an experiential course on personality. The only prerequisite for participation was completion of an introductory psychology course. This helped to ensure a broad sample of college students. Ss received a letter grade based on their participation in weekly meetings, the completion of assignments, and the completion of a written report. 


\section{General procedure}

All Ss came to weekly group meetings throughout the semester. At these meetings, they received instructions as well as completed a large number of standardized personality questionnaires. $S$ s also underwent an experiential sampling phase, where they completed multiple reports each day for a month. Finally, Ss came individually to the laboratory on several occasions for individualized testing and photography.

\section{Assessments}

Gaze avoidance assessment. Ss reported to the laboratory individually. Upon arrival they were informed that a photograph would be taken and were asked to give their informed consent, both for being photographed and for having that photo used in future research. Ss were photographed from the chest up while standing and were told to simply "Act natural". Ss were not posed for these photos whatsoever, they were simply told to act natural. Several $S$ s asked if they should smile, and they were again told to "Just act as you usually do". The photograph was taken with color slide film and was obtained using a Minolta $35 \mathrm{~mm}$ autofocus SLR camera mounted on a stationary tripod and equipped with a $50 \mathrm{~mm}$ lens.

Ss photographs were enlarged to 8.5 by 11 -inch color prints. Two raters evaluated whether or not the $S$ s faces were perpendicular with the camera. The raters laid a sheet of graphical transparency paper cross-hatched with 20 squares/inch on top of the photographs. A horizontal axis line was aligned with the center of the pupils of the eyes. A vertical axis then was drawn from the midpoint of the line connecting the two pupils to the lowermost point at the base of the chin. With this vertical axis established, the raters could quickly and reliably determine whether the face was rotated on this axis. After training, the two raters achieved $100 \%$ agreement on whether the $S$ s were or were not directly facing the camera. Gaze aversion was coded as 0 or 1 , with $0=$ gaze aversion, and $1=S$ was looking directly at the camera.

Personality assessment. Ss in both samples completed a number of standard personality questionnaires. All of the personality questionnaires employed are published in the literature and exhibit acceptable levels of reliability and validity. The $S \mathrm{~s}$ in Sample 1 completed the Mood Survey (Underwood \& Fromming, 1980), the EASI III inventory of temperament (Buss \& Plomin, 1975), the Need For Stimulation questionnaire (Herzog, Williams \& Weintraub, 1984), and the Emotional Control Questionnaire (Roger \& Nesshover, 1987).

$S$ s in Sample 2 also completed these same questionnaires. In addition, Sample 2 ss completed the Eysenck Personality Questionnairc-Revised (Eysenck, Eysenck \& Barrett, 1985), the Derogatis Physical Symptom Checklist (Derogatis \& Melisaratos, 1983), the Institute for Personality and Ability Testing (IPAT) Depression and Anxiety Scales (Krug, Scheier \& Cattell, 1976), and the Minnesota Multiphasic Personality Inventory (MMPI) (Hathaway \& McKinley, 1951).

Daily experience sampling assessment. $S$ s in each sample also underwent an experiential sampling phase, wherein they reported on a number of variables twice daily for a period of 4 consecutive weeks. Each $S$ completed one report form at midday to report on his or her experiences during the first half of that day. A second report was completed at the end of the day to report on the second half of the day. Accurate recording of daily experiences and activities was stressed by the experimenters. Reports were collected on a weekly basis. Compliance with the daily reporting task was excellent, with over $90 \%$ of the $S$ s completing $100 \%$ of the reports. The single $S$ with the worst compliance was missing only $4 \%$ of his daily reports.

Ss in both samples rated a series of mood adjectives for how much of each mood they felt during the time period. The mood adjectives were selected to represent the circumplex model of emotion (Larsen \& Diener, 1992), including emotions of pleasant and unpleasant hedonic tone at varying levels of arousal. Ss were provided with a written definition of each emotion adjective taken from Webster's New Collegiate Dictionary. The response scale went from 0 (not at all) to 6 (felt this emotion extremely much). Emotion ratings were averaged over all the occasions of observation to result in an aggregate measure of how much each $S$ experienced each emotion, on average, over the recording month.

$S$ s in both samples also used a checklist to report the occurrence of a variety of physical symptoms. For Sample 1 Ss the physical symptoms included headaches, trouble concentrating, runny nose, 
stomach aches, muscle aches, sore throat or coughs, backaches, and feeling jittery or weak. Sample $2 \mathrm{Ss}$ indicated the degree to which they had trouble getting to sleep and staying asleep the night before, using a response scale of $0=$ not at all, and $4=$ extremely. In addition, Sample $2 S$ s reported how much they had eaten during the time period, with $0=$ nothing at all, to $4=$ more than a normal meal. Sample $2 S$ s also completed a symptom checklist that included the same symptoms as for Sample 1, plus shortness of breath, temper outburst, and hot or cold flushes. For both samples, the responses to the daily symptom assessments were aggregated to total symptom scores, representing the total number of times each symptom was reported during the month of daily recording.

Observer ratings of photographs. The photographs of each sample were rated by groups of raters along various personality and evaluative dimensions. Sample $1 \mathrm{Ss}$ were rated by a group of 37 college student raters (from a different university), who participated in exchange for credit toward their grade in an introductory psychology course. Based on the photographs, the raters assessed the $S$ s on the following dimensions: Attractive-Unattractive, Happy-Unhappy, Active-Sluggish, Extroverted-Introverted, Conscientiousness-Unreliable, Agreeable-Disagreeable, Emotionally Unstable-Emotionally Stable, and Intelligent-Unintelligent. The raters used a bipolar rating scale to rate each subject's photograph, with +4 defining one pole, -4 defining the other, and 0 defining the midpoint. The ratings were standardized across $S$ s in Sample 1.

Sample $2 S$ s were rated by a group of 58 raters, who assessed the $S$ s on the same characteristics as Sample 1, with the exception of Intelligence, which was not rated on Sample $2 S$ s. The ratings for Sample $2 S$ s were similarly standardized within the sample.

A third group of 15 raters evaluated both samples of $S \mathrm{~s}$ on two dimensions: was the person smiling or frowning ( +4 for full smile, -4 for full frown), and "How genuine or real does this person's expression appear?", where $-4=$ completely false or fake, and $+4=$ completely genuine or real.

\section{RESULTS}

\section{A note on statistical power}

Although our study involves two independent samples of $S \mathrm{~s}$, and several independent sets of raters, none of our samples exceed $N=41$ when analyses are broken down by sex of subject. At the risk of increasing Type I error slightly, we will consider the results in terms of effect size, in addition to implementing standard statistical interpretation logic. A correlation coefficient can be considered an effect size in and of itself. For a bivariate correlation coefficient, an effect size provides information about the magnitude of the relationship between two variables in standard deviation units. An effect size is therefore free of sample size limitations that can obscure important results in a standard statistical interpretational framework. According to Cohen (1988), correlations between 0.10 and 0.29 represent small effect sizes, correlations between 0.30 and 0.49 represent medium effect sizes, and correlations between 0.5 and 1.0 represent large effect sizes. Correlations representing medium or large effect sizes are italicized in all tables.

We will present the results of Samples 1 and 2 in Tables 1 and 2 respectively, and note replications where they occur. We will also analyze the data separately by sex, as there are reasons to believe that turning the head may have a different meaning when done by a female than by a male (Simpson, Gangestead \& Biek, 1993). We did, in fact, find that women exhibited more gaze aversion than men. Across the two samples, $52 \%$ of the women looked askew at the camera, whereas only $31 \%$ of the men exhibited an indirect posture $(\chi=14.89, P<0.01)$. Because of this difference in base rates, and due to the possible psychological differences associated with the gender of the $S$ (discussed more fully below), we present the results separately for each sex.

Men in both samples who were gaze avoidant scored as less emotionally variable on the Mood Survey than those men who looked directly at the camera. Gaze avoidant men also reported fewer and smaller mood changes from day to day. Gaze avoidant men in Sample 1 scored lower on the EASI III measure of generally emotionality, a finding that replicated with a small effect size in Sample 2. In looking at some of the subscales of the EASI III, it was found that the gaze avoidant men were lower on sensation seeking scale ( $r$ 's $=0.45$ and 0.15 for Samples 1 and 2 ) and higher in inhibition ( $r$ 's $=-0.22$ and -0.57 for Samples 1 and 2 ). Men who were gaze avoidant also appeared 
Tabie 2. Correlations between facial orientation with psychological/personality, emotional, and physiological variables, with participation in various activities. and with observe-ratings of photographs: Sample 2

\begin{tabular}{|c|c|c|c|}
\hline & \multicolumn{3}{|c|}{ Facial orientation ${ }^{3}$} \\
\hline & Women & Men & Total \\
\hline \multicolumn{4}{|l|}{ Personality/psychological variables } \\
\hline MS-Hedonic level & -0.12 & 0.06 & -0.03 \\
\hline MS-Hedonic variability & -0.32 & $0.48^{*}$ & 0.00 \\
\hline EASI 3-Emotionality & 0.29 & 0.24 & 0.25 \\
\hline EASI 3-Activity & 0.13 & -0.05 & 0.09 \\
\hline EASI 3-Sociability & 0.02 & 0.06 & 0.09 \\
\hline EASI 3-Impulsivity & -0.33 & 0.07 & -0.18 \\
\hline SCL-Somaticization & 0.03 & -0.29 & -0.04 \\
\hline SCL-Obsessiveness & $0.50^{*}$ & $-0.7 I^{* *}$ & 0.04 \\
\hline SCL-Interpersonal sensitivity & 0.34 & -0.24 & 0.08 \\
\hline SCL-Depression & 0.37 & $-0.56^{*}$ & 0.01 \\
\hline SCL-Anxiety & 0.16 & -0.47 & -0.06 \\
\hline IPAT-Depression & -0.11 & $-0.71^{* *}$ & -0.28 \\
\hline IPAT-Apprehension & 0.22 & $-0.50^{*}$ & -0.03 \\
\hline IPAT--Tension & 0.21 & $-0.53^{*}$ & -0.09 \\
\hline IPAT-Poor emotional control & 0.05 & -0.42 & -0.12 \\
\hline IPAT Emotional instability & 0.00 & -0.38 & -0.13 \\
\hline IPAT - Suspiciousness & $0.6 I^{* * *}$ & 0.17 & $0.47^{* *}$ \\
\hline Need for stimulation & 0.13 & 0.05 & 0.14 \\
\hline ECQ-Rehearsal & $0.41^{*}$ & -0.25 & 0.14 \\
\hline ECQ-Emotional inhibition & -0.04 & -0.33 & -0.10 \\
\hline $\mathrm{ECQ}$-Anger control & 0.24 & 0.26 & 0.17 \\
\hline ECQ Benign control & 0.26 & 0.14 & 0.20 \\
\hline EPQ-R-Psychoticism & -0.23 & 0.17 & -0.10 \\
\hline EPQ-R-Extraversion & 0.03 & 0.20 & 0.09 \\
\hline EPQ-R-Neuroticism & 0.15 & 0.37 & 0.11 \\
\hline EPQ-R-Lie & -0.25 & -0.23 & -0.24 \\
\hline \multicolumn{4}{|l|}{ MMPI scales } \\
\hline L-Denial of common faults & -0.21 & -0.20 & -0.16 \\
\hline F-Invalidity & 0.01 & -0.19 & -0.05 \\
\hline $\mathrm{K}$-Defensiveness & 0.14 & -0.10 & -0.06 \\
\hline HS-Hypochondirasis & -0.20 & -0.29 & -0.16 \\
\hline D-Depression & 0.04 & -0.30 & -0.09 \\
\hline HY-Hysteria & $-0.39 *$ & -0.06 & -0.14 \\
\hline PD-Psychopathic deviance & -0.32 & $-0.48^{*}$ & $-0.35^{*}$ \\
\hline MF-Femininity & -0.36 & 0.01 & -0.10 \\
\hline PA-Paranoia & 0.28 & -0.22 & 0.09 \\
\hline PT-Psychasthenia & -0.11 & -0.38 & -0.16 \\
\hline SC-Schizophrenia & -0.03 & -0.22 & -0.06 \\
\hline MA-Mania & -0.07 & -0.23 & -0.09 \\
\hline SI Social introversion & 0.21 & -0.16 & 0.06 \\
\hline \multicolumn{4}{|c|}{ Emotions experienced, mean over 2 months } \\
\hline Lonely & 0.27 & $-0.65^{* *}$ & -0.11 \\
\hline Pleased & 0.28 & -0.24 & $0.06^{*}$ \\
\hline Calm & 0.15 & -0.21 & -0.01 \\
\hline Relaxed & 0.16 & -0.21 & -0.01 \\
\hline Composed negative affect & $0.40^{*}$ & -0.45 & 0.12 \\
\hline \multicolumn{4}{|l|}{ Physiological complaints/events } \\
\hline Runny or congestion & 0.18 & 0.35 & 0.25 \\
\hline Sore throat & 0.17 & 0.38 & 0.28 \\
\hline Nervousness & 0.33 & -0.08 & 0.17 \\
\hline Poor Appetite & 0.27 & $-0.56^{*}$ & -0.03 \\
\hline Crying/urge to cry & $0.47^{*}$ & $-0.56^{*}$ & 0.12 \\
\hline Temper outburst & $0.39^{*}$ & 0.08 & 0.26 \\
\hline Blurred vision & 0.35 & $-0.52^{*}$ & -0.06 \\
\hline Trembling/shaking hands & $0.45^{*}$ & -0.32 & 0.12 \\
\hline Trouble getting to sleep ${ }^{b}$ & -0.09 & $-0.52^{*}$ & -0.23 \\
\hline \multicolumn{4}{|l|}{ Observer-ratings from photographs } \\
\hline Happiness & 0.30 & 0.17 & 0.18 \\
\hline Attractiveness & 0.12 & -0.30 & -0.07 \\
\hline Activity level & 0.20 & -0.21 & 0.12 \\
\hline Extraversion & 0.28 & 0.01 & 0.12 \\
\hline Conscientiousness & 0.06 & -0.22 & -0.13 \\
\hline Agreeableness & 0.35 & 0.07 & 0.15 \\
\hline Emotional stability & 0.17 & -0.01 & 0.10 \\
\hline Smiling & 0.28 & 0.25 & 0.17 \\
\hline Genuineness of expression & -0.01 & 0.09 & 0.00 \\
\hline
\end{tabular}

a Facial orientation in photograph is scored as $I$ if $S$ s head is square with camera, and as 0 if $S$ s head is turned to any degree, left or right.

'Trouble getting to sleep is reported as a mean over the 2 -month study period.

Note. $N($ Women $)=26 ; N($ Men $)=18$

Note. According to Cohen (1988), correlations between 0.10 and 0.29 represent small effect sizes, correlations between 0.30 and 0.49 represent medium effect sizes, and correlations equal to or greater than 0.50 represent large effect sizes. Correlations representing medium or large effect sizes are in italics.

$* P \leqslant 0.05: * * P \leqslant 0.01, * * P \leqslant 0.001$. 
being emotionally inhibited and controlled, with the outward appearance of emotional stability. However, there is an underlying cluster of depressive and anxious symptoms, including self-reports of apprehension, tension, and a variety of somatic and psychological symptoms, including obsessiveness, trouble sleeping, and poor appetite. The MMPI correlations suggest that, while the gaze avoidant males are somewhat high in psychopathy, they are also ruminative, agitated, depressed, and have many physical complaints. The notion of the 'over-controlled' personality might describe this personality portrait (e.g. Arnold, Fleming \& Bell, 1979; Gudjonsson, Petursson \& Skulason, 1991). The 'inner' nature of the gaze avoidant male's distress is attested to by the lack of social judgment correlates. Other than a modest correlation with rated attractiveness in one sample (which did not replicate), there were no consensual character attributions made toward the gaze avoidant males by our raters. In other words, even though the gaze avoidant males were more distressed, anxious, compulsive, and inhibited, they were not rated by the judges as appearing psychologically different from the non-avoidant subjects.

With regard to women, the correlates of gaze aversion are quite different from the male results. Gaze avoidant women had none of the somatic symptoms that distinguished the gaze avoidant men. In fact, gaze avoidant women reported fewer of certain symptoms (less nervousness, fewer temper outburst, less trembling) than the more direct women. Gaze avoidant women also scored lower on the Hopkins symptom scales for depression, sensitivity, and obsessiveness. Gaze avoidant women tended to be more relaxed, pleased, and calm than the women with direct gazes.

On the EPQ-R there was a tendency for gaze avoidant women to score higher on the Psychoticism and Lie scales, albeit only with modest correlations. On the MMPI, gaze avoidant women appeared to be more traditionally feminine, endorsing stereotyped sex role behaviors and attitudes. They also scored higher on hysteria, implying a degree of suggestibility, egocentricism, and demandingness. And finally, gaze avoidant women scored higher on the Psychopathic Deviance scale, implying that they have some sociopathic tendencies, or at least that they care little about the welfare of others. On one of the MMPI validity scales, there is some indication that gaze avoidant women engage in denial, a finding that is consistent with the EPQ-R correlations with the Lie and Psychoticism scales.

Compared to men, the psychological portrait of the gaze avoidant woman is that she displays relatively more psychological health and good adjustment, at least in terms of not having the correlations with anxiety, depression, and psychosomatic disturbances found in males. Another difference in the gaze avoidant correlates for women is the strong correlation with psychopathy (both MMPI PD scale and the EPQ-R P scale), and the correlations with hysteria and traditional femininity.

Another sex difference in the correlates of gaze aversion was that observer ratings of gaze avoidant women all go in the negative direction. That is, gaze avoidant women were viewed by others as less happy and less agreeable (in both samples), and as less active, less extraverted and outgoing, less conscientious, and less stable (all moderate correlations in Sample 1, and small correlations in Sample 2). A woman who averts her gaze apparently evokes a whole cluster of negative social evaluations. There was even a tendency (in Sample $1 S \mathrm{~s}$ ) for gaze avoidant women to be rated as less intelligent and less genuine. In both samples, gaze avoidant women were smiling less.

\section{DISCUSSION}

One aspect of our findings on gaze aversion is that women engage in this behavior significantly more often than men. This sex difference in facial turning has been noted by several other researchers, including those studying people in naturalistic settings (Kendon \& Ferber, 1973) as well as researchers examining static photos of people, as in school photo albums (Mills, 1984; Ragan, 1982). Why do more women than men display gaze aversion? Some researchers have argued that head turning is a sign of submissiveness or ingratiation (e.g. Goffman, 1976; Ragan, 1982), such researchers argue that women are constrained by social roles to be less powerful than men. Women are made submissive by cultural pressures, so this theory goes, and signal their submissiveness nonverbally through gaze aversion. Research directly testing the relation between head tilting and ratings of perceived submissiveness, however, has failed to find this association (Halberstadt \& Saitla, 1987). Moreover, in the present study we found no evidence to suggest that gaze aversion 
was associated with submissiveness. If anything, gaze avoidant women endorsed those personality characteristics suggesting they are rebellious and demanding, have feminine interests but with a mix of psychopathy.

Other rcscarchers have suggested that head turning or canting in women is a non-verbal expression of sexual availability (Simpson et al., 1993). That is, head turning or canting may be a flirtatious gesture designed to communicate sexual interest. We did not collect data directly relevant to testing this notion, such as ratings of sexuality. Instead, we found that gaze avoidant women were rated as less attractive, less agreeable, and less happy than the non-avoidant women. Gaze avoidant women were also less likely to be smiling and, at least in Sample 1, were rated as less 'genuine' than the women who looked directly at the camera. Our findings are thus not consistent with the idea that head turning in women is associated with the social judgments of flirtatiousness.

It may be that there are many varieties of head turning. There is turning the face away from perpendicular to the observer/camera. This is precisely what we measured. This head turning may be accompanied by eye contact or no eye contact, and this secondary behavior may modify the social meaning of the gesture. The eyes may be diverted downward, which may be related to submissiveness (Halberstadt \& Saitta, 1987), or the eyes may be diverted upward or sideways, which may communicate disinterest or even boredom. The head turn may or may not be accompanied with a smile, which might be another way to modify the communicative meaning of this gesture. In addition to turning on the perpendicular horizontal axis, the head may be tilted on its vertical axis as well. This may be the variety of face turning most related to the impression of flirtatiousness in women (Simpson et al., 1993). One limitation of the present study is that we did not make subtle distinctions between varieties of head turnings. Our sample sizes were simply too small to further divide the group of $S \mathrm{~s}$ who were turning their faces away from the camera into those displaying different sub-types of facial turning (with and without vertical canting, smiling, eye-contact, etc.). Future researchers may find it profitable to investigate sub-types of facial turning behavior.

In our samples, we can conclude that women and, to some extent, men who averted their gaze are higher on psychopathic deviance and psychoticism than $S \mathrm{~s}$ who looked directly at the camera. As such, there may be a kernel of truth to the notion that a person who doesn't "look you in the eye" is not to be trusted. We would expect the sociopathic tendencies associated with gaze aversion to show up in negative social judgments made by raters of the photographs. What is interesting is that the negative social evaluations were associated with gaze aversion only for the women $S$ s. That is, the gaze avoidant women were rated negatively on most attributes (as being disagreeable, unreliable, unstable, unattractive, etc.) whereas the raters made no such unsavory attributions toward the gaze avoidant male $S$ s.

How might we explain these sex differences in the social judgment correlates of facial turning? One explanation might be that the women and men differ in the magnitude of this facial expression. We know that women displayed facial turning more frequently than men. It could also be that women made more of a turn away from center as well, resulting in a 'larger' expression. This seems plausible, given that women are generally more expressive than men when it comes to facial displays of emotion. Our coding scheme, while highly sensitive to facial turning (accurate to one-twentieth of an inch away from perpendicular), was nevertheless a dichotomous code. We did not measure the actual magnitude of facial turning, only its presence or absence. Future researchers might want to quantify the degree of facial turning, and examine whether these scores are monotonically related to the kinds of social judgments examined her and in related studies.

A second plausible explanation for our obtained sex differences in the social judgments of facial turning has to do with the thresholds that people have for making those judgments. That is, it could be that people are more ready to attribute negative characteristics to others when those others are gaze avoidant and female, than when they are gaze avoidant and male. There may be something in our evolutionary history that prompts us to feel unfavorably toward females who avoid direct facial exposure. Do we have more to loose when a woman is indirect or misleading with their facial expressions? When women appear to be hiding something, is that something socially dangerous? Our self-report results suggest that gaze avoidant women are, in fact, higher in psychopathic deviance, higher in hysterical tendencies, and endorse traditional feminine ideals, including the notion that women should be seductive and desirable.

Gaze avoidance in males was more related to self-reports of emotional inhibition, depression and 
anxiety, and somatic and psychological symptoms. This cluster of traits is similar to the 'overcontrolled' personality (Gudjonsson et al. 1991), where a person controls and 'internalizes' their emotions rather than 'externalizing' them in the form of behaviors that release tension, e.g. arguing, abusing alcohol. The personality correlates of gaze avoidance in males suggest that they rarely exhibit strong emotions. Instead, they have trouble sleeping, have appetite problems, and obsess. In some respects, the gaze avoidant male also has something to hide. He is not being deceptive about something that is socially dangerous, however, as might be the case with gaze avoidant women. Instead, he does not want people to look directly into his eyes and sense his anxiety and inner doubts and tensions. Perhaps the lack of negative social judgments associated with gaze aversion in males is due to the fact that, in males, gaze avoidance is not a signal that others should be wary. Instead, if gaze avoidance in males is a cue at all, it is most likely a cue of low selfconfidence, anxiety, and inhibition. We are reminded of that social game, popular among adolescent males, often called "stare down". This is where two persons lock eye contact, and stare directly at each other until one can take it no longer and looks away.

Admittedly, much of our discussion has been speculative. Research on facial expressions in general documents many sex differences, and many researchers have offered speculation as to the source of those differences. We have documented another expressive difference between men and women, and offer some speculation as to its source and function. Not only do women and men differ in the frequency of facial turning, they also differ in the correlates (both personality and social judgments) of this gesture. This makes it a particularly interesting sex difference in social behavior, and so may be informed by a functional analysis, which we speculate on above. Future researchers might examine some of the questions raised in this discussion, and might benefit from the suggestions we made for quantifying the degree of facial turning, as well as subtyping facial turning behaviors. In addition, if social judgments are studied, it might be useful to have those judgments broken down by sex of rater, as this may be an important factor in the communicative impact of facial expressions, That is, there may be a sex effect for the perceiver of facial expression as well as for the expressor.

Acknowledgements--Preparation of this manuscript was supported, in part, by Research Scientist Development Award KO1MH00704 and grant RO1-MH42057 from the National Institute of Mental Health to Randy J. Larsen and by a Jacob K. Javits Graduate Fellowship to Todd K. Shackelford. The authors wish to thank Stephen Owens and Jody Rondall for help with facial coding and data entry.

\section{REFERENCES}

Adrien, J. L., Lenoir, P., Martineau, J. \& Perrot, A. (1993). Blind ratings of early symptoms of autism based upon family home movies. Journal of the American Academy of Child and Adolescent Psychiatry, 32, 617-626.

Anolli, L. \& Lambiase, L. (1990). The role of eye conlact in conversation. Giornale Italiano di Psicologia. 17, $27-58$.

Argyle, M. \& Cook, M. (1976). Gaze and mutual gaze. Cambridge, MA: Cambridge University Press.

Argyle, M., Henderson, M.. Bond, M. \& Iizuka, Y. (1986). Cross-cultural variations in relationship rules. International Journal of Psychology, 21, 287-315.

Arnold, L., Fleming, R.\& Bell, V. (1979). The man who became angry once: A study of overcontrolled hostility. Canadian Journal of Psychiatrv. 24, 762-766.

Baron-Cohen, S. (1994). How to build a baby that can read minds: Cognitive mechanisms in mindreading. Current Psychology of Cognition, 13, 513-552.

Bond, C. F., Omar, A.. Mahmoud. A. \& Bonser, R. N. (1990). Lie detection across cultures. Journal of Nonterbal Behavior, 14. 189-204.

Buitelaar, J. K., Van Engeland, H., de Kogel, K. H. \& de Vries, H. (1991). Differences in the structure of social behaviour of autistic children and non-autistic retarded controls. Journal of Child Psychology and Psychiatry and Allied Disciplines. 32. $995-1015$

Burger, J., Gochfeld, M. \& Murray, B. G. (1992). Risk discrimination of eye contact and directness of approach in black iguanas (Ctenosaura similis). Journal of Comparative Psychology, 106, 97-101.

Buss. A. H. \& Plomin, R. (1975). A temperature theory of personality. New York: Wiley.

Campbell, R.. Heywood, C. A., Cowey, A. \& Regard, M. (1990). Sensitivity to eye gaze in prosopagnosic patients and monkeys with superior temporal sulcus ablation. Neuropsychologia, 28, 1123-1142.

Cohen, J. (1988). Statistical power analysis for the behavioral sciences (2nd edn). Hillsdale, NJ: Lawrence Erlbaum.

D'Alessio, M. (1990). Evolution of mother-neonatal interaction during lactation. Eta evolutiva, 36, 93-99.

Dequardo, J. R. \& Tandon, R. (1994). Index of affective flattening derived from examination of home movies of preschizophrenic individuals. American Journal of Psychiatry, 151, 1717.

Derogatis, L. R. \& Melisaratos, N. (1983). The Brief Symptom Inventory: An introductory report. Psychological Medicine, $13,595-605$.

Droney, J. M. \& Brooks, C. I. (1993). Attributions of self-esteem as a function of duration of eye contact. Journal of Social Psychology, 133, 715-722. 
Duranti, A. (1992). Language and bodies in social space: Samoan ceremonial greetings. American Anthropologist, 94, 657691.

Eysenck, S. B., Eysenck, H. J. \& Barrett, P. (1985). A revised version of the Psychoticism scale. Personality \& Individual Differences, 6, 21-29.

Farabee, D. J., Holcom, M. L., Ramsey, S. L. \& Cole, S. G. (1993). Social anxiety and speaker gaze in a persuasive atmosphere. Journal of Research in Personality, 27, 365-376.

Goffman, E. (1976). Gender advertisements. Studies in the Anthropology of Visual Communication, 3, 69-154.

Gudjonsson, G. H.. Petursson, H. S. \& Skulason, S. (1991). Overcontrolled hostility among prisoners and its relationship denial and personality scores. Personality and Individual Differences, 12, 17-20.

Halberstadt, A. G. \& Saitta, M. B. (1987). Gender, nonverbal behavior, and perceived dominance: A test of the theory. Journal of Personality and Social Psychology, 53, 257-272.

Hathaway, S. R. \& McKinley, J. D. (1951). The Minnesota Multiphasic Personalizy Inventory, revised. New York: Psychological Corporation.

Herzog, T. R., Williams, R. T. \& Weintraub, D. (1984). Meanwhile, back at personality ranch: The augmenters and reducers ride again. Journal of Personality and Social Psychology, 48, 1342-1352.

Iizuka, Y. (1992a). Extraversion, introversion, and visual interaction. Perceptual and Motor Skills, 74, 43-59.

Iizuka, Y. (1992b). Evaluation of gaze pairs by female observers. Japanese Journal of Experimental Social Psychology, 31 , $231-239$.

lizuka, Y. (1994). Gaze during speaking as related to shyness. Perceptual and Motor Skills, 78, 1259-1264.

Kalma, A. (1992). Gazing in triads: A powerful signal in floor apportionment. British Journal of Social Psychology, 31, 2139.

Keller, H. \& Zach, U. (1993). Developmental consequences of early eye contact behaviour. Acta Paedopsychiatrica: International Journal of Child and Adolescent Psychiatry, 56, 31-36.

Kendon, A. \& Ferber, A. (1973). A description of some human greetings. In Michael, R. P. \& Crook, H. H. (Eds) Comparative ecology and behavior of primates: Proceedings of a conference held at the zoological society, London, November, 1971. New York: Academic Press.

Kolb, B. \& Whishaw, I. Q. (1990). Fundamentals of human neuropsychology. New York: W. H. Freeman.

Krug, S. E., Schieier, I. H. \& Cattell, R. B. (1976). Handbook for the IPAT Anxiety Scale. Champaign, IL: Institute for Personality and Ability Testing.

Larsen, R. J. \& Diener, E. (1992). Promises and problems with the circumplex model of emotion. In Clark, M. S. (Ed.) Review of personality and social psychology (Vol. 13, pp. 25-59). Newbury Park, CA: Sage.

Linnankoski, I., Gronroos, M. \& Pertovaara, A. (1993). Eye contact as a trigger of male sexual arousal in stump-tailed macaques (Macaca arctoides). Folia Primatologica, 60, 181-184.

Mills, J. (1984). Self-posed behaviors of females and males in photographs. Sex Roles, 10, 633-637.

Ragan, J. M. (1982). Gender displays in portrait photographs. Sex Roles, 8, $33-43$.

Roger, D. \& Neshover, W. (1987). The construction and preliminary validation of a scale for measuring emotional control. Personality and Individual Differences, 8, 527-534.

Sherrard, C. (1993). Six principles for developmental communication: Silent-film montage and adult-infant interaction. Language and Communication, 13, 163-168.

Simpson, J. A., Gangestad, S. W. \& Biek. M. (1993). Personality and nonverbal social behavior: An ethological perspective of relationship initiation. Journal of Experimental Social Psychology, 29, 434-46I.

Underwood, B. \& Froming. W. J. (1980). The Mood Survey: A personality measure of happy and sad moods. Journal of Personality Assessment, 44, 404-414.

Volkmar, F. R. \& Mayes, L. C. (1990). Gaze behavior in autism. Development and Psychopathology, 2, 61-69.

de Waal F (1982). Chimpanzee politics. New York: Harper \& Row.

Walker, E. \& Lewine, R. J. (1990). Prediction of adult-onset schizophrenia from childhood home movies of the patients. American Journal of Psychiatry, 147, 1052-1056.

Winkel, F. W. \& Vrij, A. (1990). Interaction and impression formation in a cross-cultural dyad: Frequency and meaning of culturally determined gaze behaviour in a police interview-setting. Social Behaviour, 5, 335-350. 